\title{
Incidence, Mortality, and Predictive Factors of Hepatocellular Carcinoma in Primary Biliary Cirrhosis
}

\author{
Kenichi Hosonuma, ${ }^{1}$ Ken Sato, ${ }^{1}$ Masatoshi Yanagisawa, ${ }^{1}$ Satoru Kakizaki, ${ }^{1}$ \\ Hitoshi Takagi, ${ }^{1}$ Junko Hirato, ${ }^{2}$ and Masatomo Mori ${ }^{1}$ \\ ${ }^{1}$ Department of Medicine and Molecular Science, Gunma University Graduate School of Medicine, \\ 3-39-22 Showa-machi, Maebashi, Gunma 371-8511, Japan \\ ${ }^{2}$ Department of Pathology, Gunma University Hospital, 3-39-15 Showa-machi, \\ Maebashi, Gunma 371-8511, Japan \\ Correspondence should be addressed to Ken Sato; satoken@showa.gunma-u.ac.jp
}

Received 1 December 2012; Accepted 18 January 2013

Academic Editor: Bjørn Moum

Copyright (C) 2013 Kenichi Hosonuma et al. This is an open access article distributed under the Creative Commons Attribution License, which permits unrestricted use, distribution, and reproduction in any medium, provided the original work is properly cited.

Background. The study aims to analyze in detail the incidence, mortality using the standardized incidence ratio (SIR), and standardized mortality ratio (SMR) of hepatocellular carcinoma (HCC) in primary biliary cirrhosis (PBC), because no large case studies have focused on the detailed statistical analysis of them in Asia. Methods. The study cohorts were consecutively diagnosed at Gunma University and its affiliated hospitals. Age- or sex-specific annual cancer incidence and deaths were obtained from Japanese Cancer Registry and Death Registry as a reference for the comparison of SIR or SMR of HCC. Moreover, univariate analyses and multivariate analyses were performed to clarify predictive factors for the incidence of HCC. Results. The overall 179 patients were followed up for a median of 97 months. HCC had developed in 13 cases. SIR for HCC was 11.6 (95\% confidence interval (CI), $6.2-$ 19.8) and SMR for HCC was 11.2 (95\% CI, 5.4-20.6) in overall patients. The serum albumin levels were a predictive factor for the incidence of HCC in overall patients. Conclusions. The incidence and mortality of HCC in PBC patients were significantly higher than those in Japanese general population. PBC patients with low serum albumin levels were populations at high risk for HCC.

\section{Introduction}

Hepatocellular carcinoma (HCC) has been increasingly reported as a complication of primary biliary cirrhosis (PBC) [1-9]. Large case studies showed that the incidence of HCC $[1,2,8,9]$ in patients with PBC was significantly higher than those in the general population. However, the incidence of malignancies including HCC is still controversial [10]. On the other hand, only a few studies $[2,4,11]$ reported predictive factors for the incidence of HCC in PBC. Most of the large studies about the incidence of HCC have been performed in Europe and America, and no large case studies have focused on the detailed statistical analysis of the incidence and mortality using standardized incidence ratio (SIR) and standardized mortality ratio (SMR) of HCC in PBC in Asia. Here, we evaluated the incidence, mortality, and predictive factors for the incidence of HCC in PBC in Japan.

\section{Patients and Methods}

The present study included the patients who were consecutively diagnosed at Gunma University and its affiliated hospitals as having PBC between 1988 and 2011, and who were followed up for more than or equal to 12 months. In addition, the eligibility of the cohorts is that HCC screening interval is 6 to 12 months. We reviewed the medical charts retrospectively to evaluate these subjects and extracted patients who had developed HCC from the time of the diagnosis of PBC to the date of death, or on September 30, 2011. The study protocol was compliant to the ethical guidelines of the 1975 Declaration of Helsinki.

The criteria for the diagnosis of $\mathrm{PBC}$ were positive antimitochondrial antibody test assessed by enzyme-linked immunosorbent assay in combination with increased serum alkaline phosphatase activity and compatible liver histology. 
We confirmed histological assessment at diagnosis of PBC in all subjects. Histological findings were classified according to Scheuer's classification [12]. Symptoms of PBC were defined as pruritus, persistent fatigue (lasting greater than three months in the absence of other identifiable causes such as anemia or hypothyroidism), overt jaundice, bleeding esophageal varices, ascites, hepatic encephalopathy or persistent right hypochondrial pain in the absence of another cause such as cholelithiasis [13].

HCC was diagnosed if the following conditions were met: (1) pathological features consistent with HCC were identified by histological examination of liver tissue obtained by needle biopsy or other procedures; or (2) one or more hepatic spaceoccupying lesions were detected by ultrasonography or computed tomography and were shown to have vascular patterns typical of HCC by angiography, triple-phase spiral computed tomography, or contrast-enhanced magnetic resonance imaging. Because our study was performed using several modalities to screen HCC and the screening interval is 6 to 12 months, we assessed the comparability of the ratio of types of modalities to screen HCC, the screening intervals and the ratio of combination of modalities, and the screening intervals between the patients who had developed HCC and those who had not developed HCC. On the other hand, ursodeoxycholic acid improves prognosis in view of liver biochemistry, histological progression, the development of portal hypertension and its complications [14-18], and combination of ursodeoxycholic acid and bezafibrate [19-21] or bezafibrate alone $[21,22]$ are effective for PBC. Moreover, several observational studies [18, 23-27] have shown that the long-term outcome of PBC correlates with the biochemical response to ursodeoxycholic acid. Then, we evaluated the ratio of types of therapeutic regimen and the ratio of groups with responders or non-responders to ursodeoxycholic acid by the criteria based on Barcelona [24], Paris [25], Rotterdam [26], and Toronto definitions [27], patients who received only bezafibrate or those who received neither ursodeoxycholic acid nor bezafibrate between those who had developed HCC and those who had not developed HCC. Time to event was calculated by the intervals from the diagnosis of $\mathrm{PBC}$ to the diagnosis of HCC. Each principle physician censored the development of HCC. The analyses have been carried out with or without the period of follow-up for one year after the diagnosis of $\mathrm{PBC}$ because of reducing the impact of selection bias (the investigation of symptoms shortly after the diagnosis of PBC might increase the chance of cancer being investigated and diagnosed). Person years at the risk of follow-up in the incidence and mortality were calculated for each patient starting immediately (and one year) after the diagnosis of $\mathrm{PBC}$, and ending with HCC registration, death, or end of the follow-up period. Age- or sex-specific annual cancer incidents and deaths were obtained from Japanese Cancer Registry and Death Registry. The SIR or SMR and their 95\% confidence intervals (CI) based on the Poisson distribution were then calculated using the population of the whole Japan as the standard population because these data were not available in the Gunma prefecture.
All data about these parameters were collected in the medical charts, death certificate, and questionnaires. The predictive variables of the development of HCC were extracted from the data at the diagnosis of PBC. Although some data was missing, definitive histological diagnosis of $\mathrm{PBC}$ and complying with screening methods for HCC was requisite for registering as our study cohort. The analysis about SIR, SMR, and predictive factors was conducted for overall or female patients because the number of males was small. We assessed the associations between the incidence of HCC and laboratory and clinical variables including several variables (cigarette smoking; alcohol consumption; blood transfusion; familial history of malignancies; liver diseases; autoimmune conditions; diabetes mellitus; hypertension; hyperlipidemia, and body mass index). Continuous data are presented as median and range. For quantitive data, analyses were performed using the Mann-Whitney $U$-test for comparison of two independent groups. Differences in proportions were performed by the Fisher's exact test. The Spearman's rankcorrelation coefficient (two-tailed) was used to evaluate the correlation between the variables. Basically, variables that achieved statistical significance and marginal significance on univariate analysis were entered into multivariate analysis by the Cox proportional hazards model to identify significant independent predictive factors. $P<0.05$ was considered significant. All analyses were performed using the IBM SPSS statistical software package, version 16 (IBM Corp., Armonk, NY, USA).

\section{Results}

The clinicopathological characteristics of the study population at diagnosis of PBC were summarized in Table 1. The overall sample included 179 patients (24 males, 155 females). The follow-up period ranged from 12 to 281 months with a median of 97 months. The median age was 57 years (range 22-85). We completed follow-up of the patients without any dropout. One hundred and one patients had Scheuer's stage I, 42 had stage II, 19 had stage III, and 17 had stage IV. Of a total of 179 patients, $53(29.6 \%)$ were symptomatic at diagnosis. Anti-HCV antibody and hepatitis B virus surface antigen were tested in all patients. No patients were positive for anti-HCV antibody and hepatitis B virus surface antigen. Although hepatitis B virus core antibody was not measured in some patients, all patients who had developed HCC were negative for the antibody. The ratio of types of the modalities to screen HCC and the follow-up period were not significantly different between the patients who had developed HCC and those who had not developed HCC in overall or female patients (Table 2). In addition, the ratio of combination of the modalities and the follow-up period such as biannual CT were not significantly different between them in overall or female patients. Furthermore regarding the types of therapeutic regimen and the ratio of groups with responders or non-responders to ursodeoxycholic acid defined by the criteria as mentioned above [24-27], patients received only bezafibrate or those who received neither ursodeoxycholic acid nor bezafibrate were not significantly different between those who had developed HCC and those 
TABLE 1: Clinicopathological characteristics of the study population.

\begin{tabular}{|c|c|c|c|}
\hline Parameters & Male & Female & Overall \\
\hline Number of patients & 24 & 155 & 179 \\
\hline Age of diagnosis of primary biliary cirrhosis (years) ${ }^{*}$ & $55.5(22-75)$ & $57(24-85)$ & $57(22-85)$ \\
\hline Duration of follow-up (months) ${ }^{* \dagger}$ & $96.5(27-218)$ & $97(12-281)$ & $97(12-281)$ \\
\hline Number of patients with Scheuer's stage (I/II/III/IV) & $13 / 5 / 2 / 4$ & $88 / 37 / 17 / 13$ & $101 / 42 / 19 / 17$ \\
\hline Brinkman index ${ }^{*}$ & $400(0-1575)$ & $0(0-940)$ & $0(0-1575)$ \\
\hline Number of patients with a history of blood transfusion & 3 & 20 & 23 \\
\hline Number of patients with a history of drinking & 4 & 7 & 11 \\
\hline Number of symptomatic patients & 4 & 49 & 53 \\
\hline Pruritus & 1 & 36 & 37 \\
\hline Jaundice & 2 & 7 & 9 \\
\hline Ascites & 0 & 3 & 3 \\
\hline Varices & 2 & 15 & 17 \\
\hline \multicolumn{4}{|l|}{ Biochemical data } \\
\hline $\operatorname{AST}(\mathrm{IU} / \mathrm{L})^{*}$ & $59.5(26-140)$ & $46.5(16-258)$ & $49(16-258)$ \\
\hline $\operatorname{ALT}(\mathrm{IU} / \mathrm{L})^{*}$ & $72(17-233)$ & $41(10-329)$ & $46(10-329)$ \\
\hline $\operatorname{ALP}(1 \mathrm{U} / \mathrm{L})^{*}$ & $652(94-2113)$ & $501.5(112-2985)$ & $535.5(94-2985)$ \\
\hline$\gamma$-GTP $(\mathrm{IU} / \mathrm{L})^{*}$ & $350.5(75-1349)$ & $164(13-1004)$ & $173.5(13-1349)$ \\
\hline $\operatorname{Alb}(\mathrm{g} / \mathrm{dL})^{*}$ & $4.2(2.4-5.1)$ & $4.2(1.9-5.0)$ & $4.2(1.9-5.1)$ \\
\hline T-Bil $(\mathrm{mg} / \mathrm{dL})^{*}$ & $0.8(0.3-3.9)$ & $0.7(0.1-6.3)$ & $0.7(0.1-6.3)$ \\
\hline $\operatorname{IgG}(\mathrm{mg} / \mathrm{dL})^{*}$ & $1660(1194-3092)$ & $1676(778-4532)$ & $1660(778-4532)$ \\
\hline $\operatorname{IgM}(\mathrm{mg} / \mathrm{dL})^{*}$ & $482(72-1465)$ & $346(48-1660)$ & $352.5(48-1660)$ \\
\hline Plt $\left(\times 10^{4} / \mu \mathrm{L}\right)^{*}$ & $24.4(8.7-58.1)$ & $20.7(5.3-48.6)$ & $20.9(5.3-58.1)$ \\
\hline $\mathrm{PT}(\%)^{*}$ & $105.0(78.0-126.5)$ & $100.0(44.0-139.0)$ & $100.0(44.0-139.0)$ \\
\hline Number of patients with medications (UDCA/BF/UDCA + BF/none) & $17 / 0 / 7 / 0$ & $127 / 1 / 22 / 5$ & $144 / 1 / 29 / 5$ \\
\hline Number of deaths during follow-up & 4 & 27 & 31 \\
\hline
\end{tabular}

who had not developed HCC in overall or female patients (Table 2).

During follow-up, HCC had developed in 13 cases (Table 2). The median age of the patients at the diagnosis of HCC was 68 years (range, 56-80). The median age of the patients at the diagnosis of PBC was 63 years (range, 46-77). The median period from the time of the diagnosis of $\mathrm{PBC}$ to the time of the diagnosis of HCC was 76 months (range, 9-180). All of the HCC was diagnosed through screening alone, but not a workup of new symptoms. The diameter of all detected HCC is less than or equal to $3 \mathrm{~cm}$. Of 13 HCC cases, histological examination of the background liver was performed in five cases and showed that one had Scheuer's I, one had Scheuer's II, one had Scheuer's III, and two had Scheuer's IV. Five cases had TNM stage I, six had stage II, and two had stage III at the HCC diagnosis. As for the treatments, operation, transcatheter arterial chemoembolization, and/or radiofrequency ablation and microwave coagulation therapy could be applied to ten cases, and three cases were incurable because of their poor liver function.

The total number of person years at the risk of follow-up in the incidence calculation was 1,608 when using all incident HCC and 1,447 when omitting the first year of follow-up after PBC diagnosis in overall patients. Table 3 shows the SIR of HCC. The SIR of HCC in overall or female patients was significantly higher than that in general population whether the first year of follow-up after PBC diagnosis was excluded or not.

The total number of person years at the risk of followup in the mortality calculation was 1,776 when including all deaths. The length of follow-up for subjects varied between 12 and 281 months with a median of 97 months. The primary cause of death includes 13 cases of malignancy death (nine cases of HCC, one case of lung cancer, one case of malignant lymphoma, one case of uterine corpus cancer, and one case of skin cancer), nine cases of liver failure, and nine cases of other causes such as subarachnoid hemorrhage (Table 4). Table 3 gives the SMR of HCC. The SMR of HCC in overall or female patients was significantly higher than that in general population.

The overall prevalence of associated autoimmune conditions was $32.4 \%$. Sjögren's Syndrome was the most frequent association: it was present in $14.5 \%$ of cases. Thyroid disease was observed in $8.9 \%$ and rheumatoid arthritis in $8.9 \%$ of cases. Systemic sclerosis was recorded in $8.4 \%$ and systemic lupus erythematosus in $0.6 \%$ of cases.

A history of smoking (more than 10 cigarettes per day) was observed in $20.7 \%$ (14.8\% in females, $58.3 \%$ in males). A history of alcohol consumption more than $20 \mathrm{~g}$ /day in males was $16.7 \%$ and that more than $20 \mathrm{~g} /$ day in females was $4.5 \%$. 
TABLE 2: Comparison of clinicopathological characteristics between patients who had developed hepatocellular carcinoma (HCC) and those who had not developed HCC at follow-up.

\begin{tabular}{|c|c|c|c|c|c|c|}
\hline & \multicolumn{2}{|c|}{ Overall } & \multicolumn{4}{|c|}{ Female } \\
\hline & $\begin{array}{l}\text { Patients who had } \\
\text { developed HCC at } \\
\text { follow-up }\end{array}$ & $\begin{array}{l}\text { Patients who had } \\
\text { not developed } \\
\text { HCC at follow-up }\end{array}$ & $P$ & $\begin{array}{l}\text { Patients who had } \\
\text { developed HCC at } \\
\text { follow-up }\end{array}$ & $\begin{array}{l}\text { Patients who had } \\
\text { not developed } \\
\text { HCC at follow-up }\end{array}$ & $P$ \\
\hline Number of patients & 13 & 166 & & 11 & 144 & \\
\hline Number of male patients & 2 & 22 & 0.687 & NA & NA & NA \\
\hline Age of Dx of PBC (years) ${ }^{*}$ & $63(46-77)$ & $56(22-85)$ & 0.072 & $63(46-77)$ & $56(24-85)$ & 0.049 \\
\hline PBC Stage at Dx of PBC (I/II/III/IV) & $2 / 4 / 3 / 4$ & $99 / 38 / 16 / 13$ & 0.004 & $2 / 3 / 3 / 3$ & $86 / 34 / 14 / 10$ & 0.011 \\
\hline Number of symptomatic patients & 8 & 45 & 0.022 & 7 & 42 & 0.037 \\
\hline Pruritus & 4 & 33 & 0.474 & 4 & 32 & 0.282 \\
\hline Jaundice & 1 & 8 & 0.501 & 1 & 6 & 0.409 \\
\hline Ascites & 0 & 3 & 1 & 0 & 3 & 1 \\
\hline Varices & 5 & 12 & 0.003 & 4 & 11 & 0.013 \\
\hline \multicolumn{7}{|l|}{ Biochemical data } \\
\hline $\operatorname{AST}(\mathrm{IU} / \mathrm{L})^{*}$ & $78(30-231)$ & $46(16-258)$ & 0.088 & $77(30-231)$ & $46(16-258)$ & 0.15 \\
\hline $\operatorname{ALT}(\mathrm{IU} / \mathrm{L})^{*}$ & $51(22-319)$ & $45(10-329)$ & 0.644 & $47.5(22-319)$ & $41(10-329)$ & 0.761 \\
\hline $\operatorname{ALP}(\mathrm{IU} / \mathrm{L})^{*}$ & $402.5(175-1185)$ & $543.5(94-2985)$ & 0.314 & $402.5(175-1185)$ & $543.5(112-2985)$ & 0.489 \\
\hline$\gamma \mathrm{GTP}(\mathrm{IU} / \mathrm{L})^{*}$ & $174.5(16-470)$ & $173.5(13-1349)$ & 0.285 & $114(16-470)$ & $173.5(13-1004)$ & 0.181 \\
\hline $\lg G(\mathrm{mg} / \mathrm{dL})^{*}$ & $2165(1030-4532)$ & $1655(778-3900)$ & 0.021 & $2165(1030-4532)$ & $1660(778-3900)$ & 0.049 \\
\hline $\lg M(\mathrm{mg} / \mathrm{dL})^{*}$ & 405 (136-918) & $351.5(48-1660)$ & 0.364 & 359.5 (136-918) & $336(48-1660)$ & 0.352 \\
\hline $\operatorname{Alb}(\mathrm{g} / \mathrm{dL})^{*}$ & $3.7(1.9-4.6)$ & $4.2(2.0-5.1)$ & 0.016 & $4.0(1.9-4.6)$ & $4.2(2.0-5.0)$ & 0.085 \\
\hline T-Bil $(\mathrm{mg} / \mathrm{dL})^{*}$ & $0.7(0.3-2.5)$ & $0.7(0.1-6.3)$ & 0.925 & $0.7(0.3-2.5)$ & $0.7(0.1-6.3)$ & 0.888 \\
\hline $\operatorname{Plt}\left(\times 10^{4} / \mu \mathrm{L}\right)^{*}$ & $12.4(5.3-24.4)$ & $21.4(5.3-58.1)$ & 0.001 & $12.3(5.3-21.8)$ & $21.0(5.3-48.6)$ & 0.001 \\
\hline $\mathrm{PT}(\%)^{*}$ & $96.5(57.0-117.0)$ & $100.9(44.0-139.0)$ & 0.204 & $93.0(57.0-117.0)$ & $100.9(44.0-139.0)$ & 0.123 \\
\hline ANA positive & 7 & 106 & 0.658 & 6 & 98 & 0.579 \\
\hline Brinkman index ${ }^{*}$ & $0(0-400)$ & $0(0-1575)$ & 0.175 & $0(0-0)$ & $0(0-940)$ & 0.12 \\
\hline $\begin{array}{l}\text { Number of patients with a history of blood } \\
\text { transfusion }\end{array}$ & 3 & 20 & 0.415 & 3 & 17 & 0.285 \\
\hline $\begin{array}{l}\text { Number of patients with a history of } \\
\text { drinking }\end{array}$ & 0 & 11 & 1 & 0 & 7 & 1 \\
\hline $\begin{array}{l}\text { Number of patients with a familial history } \\
\text { of malignancy }\end{array}$ & 3 & 61 & 0.359 & 2 & 56 & 0.261 \\
\hline $\begin{array}{l}\text { Number of patients with a familial history } \\
\text { of liver disease }\end{array}$ & 1 & 25 & 0.764 & 0 & 24 & 0.337 \\
\hline Number of patients with DM & 2 & 17 & 0.632 & 2 & 14 & 0.317 \\
\hline Number of patients with HT & 5 & 27 & 0.059 & 4 & 22 & 0.09 \\
\hline Number of patients with HL & 3 & 40 & 1 & 3 & 34 & 0.725 \\
\hline $\begin{array}{l}\text { Number of patients with autoimmune } \\
\text { disease }\end{array}$ & 5 & 53 & 0.759 & 5 & 51 & 0.528 \\
\hline Sjogren's syndrome & 2 & 24 & 1 & 2 & 24 & 1 \\
\hline Hashimoto's disease & 3 & 15 & 0.128 & 3 & 15 & 0.12 \\
\hline Rheumatoid arthritis & 1 & 15 & 1 & 1 & 14 & 1 \\
\hline Scleroderma & 0 & 15 & 0.606 & 0 & 15 & 0.602 \\
\hline Systemic lupus erythematosus & 0 & 2 & 1 & 0 & 1 & 1 \\
\hline BMI* $^{*}$ & $24.3(13.8-27.4)$ & $22.6(14.3-33.1)$ & 0.341 & $24.3(15.8-27.4)$ & $22.6(16.0-32.5)$ & 0.536 \\
\hline
\end{tabular}


TABLe 2: Continued.

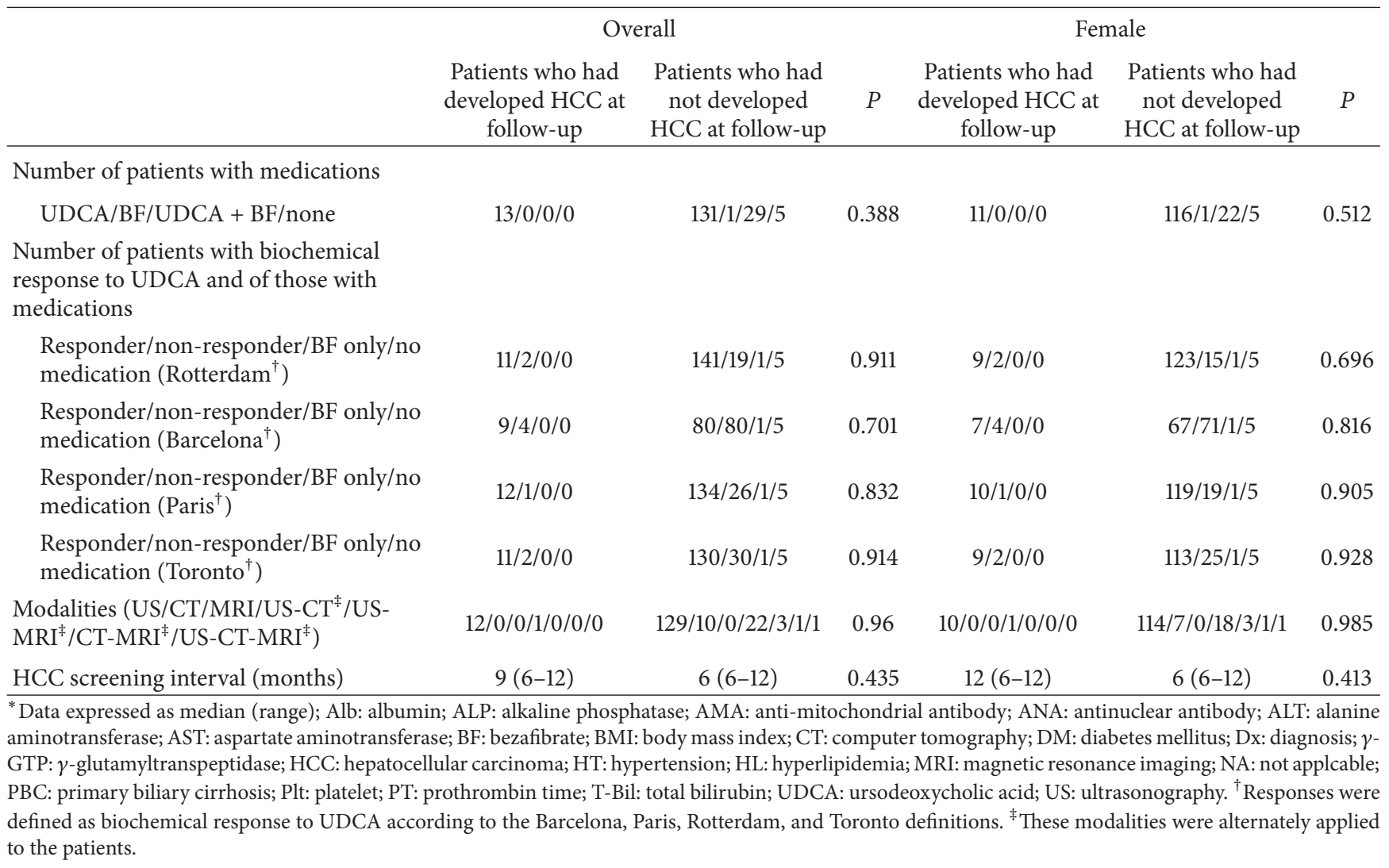

TABLE 3: Standardized incidence ratio (SIR) and standardized mortality ratio (SMR) of HCC in primary biliary cirrhosis.

\begin{tabular}{|c|c|c|c|c|}
\hline & \multicolumn{2}{|c|}{$\begin{array}{l}\text { Number of } \\
\text { incidence of HCC }\end{array}$} & \multirow[b]{2}{*}{ SIR } & \multirow[b]{2}{*}{$95 \% \mathrm{Cl}$} \\
\hline & Observed & Expected & & \\
\hline Overall & $13(12)$ & $1.1(1.0)$ & $11.6(11.5)$ & $6.2-19.8(6.0-20.2)$ \\
\hline \multirow[t]{3}{*}{ Female } & $11(10)$ & $0.5(0.5)$ & $20.4(19.8)$ & $10.2-36.5(9.5-36.4)$ \\
\hline & \multicolumn{2}{|c|}{ Deaths due to HCC } & & \\
\hline & Observed & Expected & SMR & $95 \% \mathrm{Cl}$ \\
\hline Overall & 9 & 0.9 & 11.2 & $5.4-20.6$ \\
\hline Female & 8 & 0.4 & 21.5 & $9.8-40.7$ \\
\hline
\end{tabular}

Including all years of follow up (Excluding experience in first year after diagnosis of primary biliary cirrhosis); CI: confidence interval; HCC: hepatocellular carcinoma; SIR: standardized incidence ratio; SMR: standardized mortality ratio.

The rate of obesity (body mass index; 30 or more) was $1.7 \%$. A history of blood transfusion before a PBC diagnosis was observed in $12.8 \%$ of cases. A familial history of malignancy was observed in $35.8 \%$ and familial history of liver disease in $14.5 \%$ of cases. Diabetes mellitus was observed in $10.6 \%$ of cases, hypertension in $17.9 \%$, and hyperlipidemia in $24.0 \%$ of cases.

Variables that associated with the development of HCC were analyzed by both univariate and multivariate analyses. A univariate analysis identified some variables associated with the development of HCC in overall or female patients,
TABle 4: Primary cause of death.

\begin{tabular}{lccc}
\hline Cause & Male & Female & Overall \\
& $(n)$ & $(n)$ & $(n)$ \\
\hline Malignancies & 2 & 11 & 13 \\
Hepatocellular carcinoma & 1 & 8 & 9 \\
Others & 1 & 3 & 4 \\
$\quad$ Lung cancer & 1 & & \\
$\quad$ Malignant lymphoma & & 1 & \\
$\quad$ Uterine corpus cancer & & 1 & \\
$\quad$ Skin cancer & & 1 & \\
Liver failure (nonmalignant) & 1 & 8 & 9 \\
Others & 1 & 8 & 9 \\
Subarachnoid hemorrhage & 1 & & \\
Heart failure & & 3 & \\
Pneumonia & & 1 & \\
Superior mesenteric artery thrombosis & & 1 & \\
Gastrointestinal bleeding & & 1 & \\
Sepsis & & 1 & \\
Acute heart disease & & 1 & \\
\hline Total & 4 & 27 & 31 \\
\hline
\end{tabular}

$n$ : numbers of patients.

which reached statistical significance or marginal significance. Among these variables, however, some variables were significantly correlated with a certain variable or others by the Spearman's rank-correlation coefficient. Then we used 
TABLE 5: A risk factor associated with the development of HCC. Adjusted odds ratios derived from the Cox proportional hazards model.

\begin{tabular}{lcccc}
\hline Factor & \multicolumn{2}{c}{ Overall } & \multicolumn{2}{c}{ Female } \\
& OR $(95 \% \mathrm{Cl})$ & $P$ & OR $(95 \% \mathrm{Cl})$ & $P$ \\
\hline $\begin{array}{l}\text { Serum albumin } \\
\text { levels }\end{array}$ & 0.205 & $<0.001$ & 0.228 & 0.001 \\
\hline
\end{tabular}

OR: odds ratio; $\mathrm{Cl}$ : confidence interval.

variables that were not significantly correlated one another. In the analysis of overall patients, these variables included Brinkman index (number of cigarettes per day $\times$ years of smoking), the serum albumin levels, the serum AST levels, the platelet counts, and the past history of Hashimoto's disease. A multivariate analysis identified the serum albumin levels that independently influenced the development of HCC in overall patients (Table 5). In the analysis of female patients, variables identified by the same method as mentioned above included Brinkman index, the serum albumin levels, the serum AST levels, the platelet counts, and the past history of Hashimoto's disease. A multivariate analysis identified the serum albumin levels that independently influenced the development of HCC in female patients (Table 5).

\section{Discussion}

In our study, the incidence risk of HCC was significantly increased in PBC patients in comparison to adjusted general population with or without the period of follow-up for a year after PBC diagnosis. The increased incidence risk of HCC in PBC patients was consistent with the previous crude large case study in Japan [28]. Our study is of quite value in view of the precise analysis using SIR and SMR differing from the previous study [28]. Outside Japan, the increased incidence risk of HCC in PBC patients is controversial [1, 2, 8-10]. Loof et al. [10] examined 559 patients with PBC with a mean follow-up period of 9 years in Sweden; they concluded that there was no significant increase in the occurrence of HCC in patients with PBC. However, HCC may have remained undetected in this study, because less than $50 \%$ of the deceased patients had an autopsy performed. In addition, racial difference may contribute to the incidence of HCC in PBC patients.

Among the large population-based studies, only a few studies $[2,4,11]$ examined the predictive factors for the development of HCC. Reported predictive factors were cigarette smoking [2], HCV positivity [2], older age at diagnosis [4], male sex [4], history of blood transfusion [4], and advanced histological stage [11]. The serum albumin levels were the independent predictive factor for the development of HCC and significantly correlated with symptom of $\mathrm{PBC}$, histological stage at the diagnosis of $\mathrm{PBC}$, or age at the diagnosis of $\mathrm{PBC}$ by the Spearman's rank-correlation coefficient in our study. In addition, symptomatic PBC frequently shows advanced stage of PBC [29]. Taken together, the serum albumin levels considered as an indicator of advanced PBC were selected for the predictive variable of HCC, supporting the recent study [11]. On the other hand, branchedchain amino acid is reported to increase serum albumin levels in patients with decompensated cirrhosis [30] and inhibits hepatocarcinogeneis in patients with decompensated cirrhosis with higher baseline body mass index or alphafetoprotein levels [31] and with compensated cirrhosis with lower baseline albumin levels [32]. In cirrhotic patients, total albumin levels decrease and simultaneously a ratio of oxidized albumin within total albumin increases. Antioxidant properties of oxidized albumin is reportedly weaker than those of reduced albumin [33]. Although being speculative, reduced antioxidant effect based on hypoalbuminemia and concomitant change of quality of albumin in advanced stage of PBC might be one of the reasons of hepatocarcinogensis.

Because our study includes small sample size of male study cohorts, the interpretation of contribution of male sex to incidence of HCC is limited. In our study, a history of smoking was not a significant predictive factor for the development of HCC, although relatively higher cigarette consumption in female study cohorts was found, suggesting that racial differences of the study population between the study [2] and the other studies [4, 11] including our study may be one possible reason of the difference of the predictive factors. The history of blood transfusion in our study was $12.8 \%$, comparable to $15.6 \%$ of that in a total of 119,792 study cohorts in The Japan Collaborate Cohort Study for Evaluation of Cancer Risk (JACC Study) [34] and 11.6\% in the previous study [4] that identifies the history of blood transfusion as a predictive factor for HCC development. Thus, although the exact reasons of the difference of the predictive factors between the study [4] and our study are unknown, the possible reason is that it might affect carcinogenesis by infections with occult hepatitis B virus or unknown transfusion-transmissible viruses in the earlier study [4] due to a hospital-based study.

Malignancy death was the most common and HCC occupied the bulk of malignancy death in our study. Our study found evidence for an excess mortality from HCC (SMR, 11.2; 95\% CI, 5.4-20.6), which confirms the studies in The Netherlands [35] and England [1].

The limitation of our study is that the sample size of the study cohort is relatively small, our study population derived from the hospitals may increase the SIR and the SMR, and our study population based on a local Japan area may not always reflect data derived from a nationwide register.

\section{Conclusion}

In conclusion, our detailed analysis showed that the incidence and mortality of HCC in overall PBC patients were significantly higher than those in Japanese general population. PBC patients with low serum albumin levels were populations at high risk for HCC. Although therapeutic options in HCC patients with low serum albumin levels may be limited, early detection of HCC may result in inhibition of reduction of the therapeutic options and thus improve the prognosis in some patients. Thus, regular monitoring for early detection of HCC may be a good strategy for PBC patients with low serum albumin levels. 


\section{Conflict of Interests}

The authors declare no conflict of interests.

\section{Acknowledgment}

The authors acknowledge the members of Gunma Liver Study Group for their cooperation in questionnaire survey.

\section{References}

[1] D. Howel, J. V. Metcalf, J. Gray et al., "Cancer risk in Primary biliary cirrhosis: a study in northern England," Gut, vol. 45, no. 5, pp. 756-760, 1999.

[2] A. Floreani, A. Baragiotta, V. Baldo et al., "Hepatic and extrahepatic malignancies in Primary biliary cirrhosis," Hepatology, vol. 29, no. 5, pp. 1425-1428, 1999.

[3] D. E. Jones, J. V. Metcalf, J. D. Collier et al., "Hepatocellular carcinoma in primary biliary cirrhosis and its impact on outcomes," Hepatology, vol. 26, no. 5, pp. 1138-1142, 1997.

[4] A. Shibuya, K. Tanaka, H. Miyakawa et al., "Hepatocellular carcinoma and survival in patients with primary biliary cirrhosis," Hepatology, vol. 35, no. 5, pp. 1172-1178, 2002.

[5] W. M. Melia, P. J. Johnson, J. Neuberger, S. Zaman, B. C. Portmann, and R. Williams, "Hepatocellular carcinoma in primary biliary cirrhosis: detection by $\alpha$-fetoprotein estimation," Gastroenterology, vol. 87, no. 3, pp. 660-663, 1984.

[6] Y. Nakanuma, T. Terada, K. Doishita, and A. Miwa, "Hepatocellulr carcinoma in primary biliary cirrhosis: an autopsy study," Hepatology, vol. 11, no. 6, pp. 1010-1016, 1990.

[7] N. Krasner, P. J. Johnson, B. Portmann et al., "Hepatocellular carcinoma in primary biliary cirrhosis: report of four cases," Gut, vol. 20, no. 3, pp. 255-258, 1979.

[8] H. T. Sorensen, S. Frijs, J. H. Olsen et al., "Risk of liver and other types of cancer in patients with cirrhosis: a nationwide cohort study in Denmark," Hepatology, vol. 28, no. 4, pp. 921-925, 1998.

[9] P. K. Nijhawan, T. M. Therneau, E. R. Dickson et al., "Incidence of cancer in Primary biliary cirrhosis: the Mayo experience," Hepatology, vol. 29, no. 5, pp. 1396-1398, 1999.

[10] L. Loof, H. O. Adami, P. Sparen et al., "Cancer risk in Primary biliary cirrhosis: a population-based study from Sweden," Hepatology, vol. 20, no. 1, pp. 101-104, 1994.

[11] A. Cavazza, L. Caballerí, A. Floreani et al., "Incidence, risk factors, and survival of hepatocellular carcinoma in primary biliary cirrhosis: comparative analysis from two centers," Hepatology, vol. 50, no. 4, pp. 1162-1168, 2009.

[12] P. Scheuer and J. Lefkowitch, Liver Biopsy Interpretation, WB Saunders, London, UK, 1994.

[13] M. I. Prince, A. Chetwynd, W. L. Craig et al., "Asymptomatic primary biliary cirrhosis: clinical features, prognosis, and symptom progression in a large population based cohort," Gut, vol. 53, no. 6, pp. 865-870, 2004.

[14] R. E. Poupon, B. Balkau, E. Eschwege, and R. Poupon, "A multicenter, controlled trial of ursodiol for the treatment of primary biliary cirrhosis," The New England Journal of Medicine, vol. 324, no. 22, pp. 1548-1554, 1991.

[15] P. Angulo, K. P. Batts, T. M. Therneau, R. A. Jorgensen, E. R. Dickson, and K. D. Lindor, "Long-term ursodeoxycholic acid delays histological progression in primary biliary cirrhosis," Hepatology, vol. 29, no. 3, pp. 644-647, 1999.
[16] K. D. Lindor, R. A. Jorgensen, T. M. Therneau, M. Malinchoc, and E. R. Dickson, "Ursodeoxycholic acid delays the onset of esophageal varices in primary biliary cirrhosis," Mayo Clinic Proceedings, vol. 72, no. 12, pp. 1137-1140, 1997.

[17] R. E. Poupon, K. D. Lindor, K. Cauch-Dudek, E. R. Dickson, R. Poupon, and E. J. Heathcote, "Combined analysis of randomized controlled trials of ursodeoxycholic acid in primary biliary cirrhosis," Gastroenterology, vol. 113, no. 3, pp. 884-890, 1997.

[18] C. Corpechot, O. Chazouillères, and R. Poupon, "Early primary biliary cirrhosis: biochemical response to treatment and prediction of long-term outcome," Journal of Hepatology, vol. 55, no. 6, pp. 1361-1367, 2011.

[19] Y. Takeuchi, F. Ikeda, S. Fujioka et al., "Additive improvement induced by bezafibrate in patients with primary biliary cirrhosis showing refractory response to ursodeoxycholic acid," Journal of Gastroenterology and Hepatology, vol. 26, no. 9, pp. 1395-1401, 2011.

[20] R. Hazzan and R. Tur-Kaspa, "Bezafibrate treatment of primary biliary cirrhosis following incomplete response to ursodeoxycholic acid," Journal of Clinical Gastroenterology, vol. 44, no. 5, pp. 371-373, 2010.

[21] S. Iwasaki, H. Ohira, S. Nishiguchi et al., "The efficacy of ursodeoxycholic acid and bezafibrate combination therapy for primary biliary cirrhosis: a prospective, multicenter study," Hepatology Research, vol. 38, no. 6, pp. 557-564, 2008.

[22] M. Nakamuta, M. Enjoji, K. Kotoh, N. Shimohashi, and Y. Tanabe, "Long-term fibrate treatment for PBC," Journal of Gastroenterology, vol. 40, no. 5, pp. 546-547, 2005.

[23] P. Angulo, K. D. Lindor, T. M. Therneau et al., "Utilization of the Mayo risk score in patients with primary biliary cirrhosis receiving ursodeoxycholic acid," Liver, vol. 19, no. 2, pp. 115-121, 1999.

[24] A. Pares, L. Caballeria, and J. Rodes, "Excellent long-term survival in patients with primary biliary cirrhosis and biochemical response to ursodeoxycholic acid," Gastroenterology, vol. 130, no. 3, pp. 715-720, 2006.

[25] C. Corpechot, L. Abenavoli, N. Rabahi et al., "Biochemical response to ursodeoxycholic acid and long-term prognosis in primary biliary cirrhosis," Hepatology, vol. 48 , no. 3, pp. 871$877,2008$.

[26] E. M. Kuiper, B. E. Hansen, R. A. de Vries et al., "Improved prognosis of patients with primary biliary cirrhosis that have a biochemical response to ursodeoxycholic acid," Gastroenterology, vol. 136, no. 4, pp. 1281-1287, 2009.

[27] T. Kumagi, M. Guindi, S. E. Fischer et al., "Baseline ductopenia and treatment response predict long-term histological progression in primary biliary cirrhosis," American Journal of Gastroenterology, vol. 105, no. 10, pp. 2186-2194, 2010.

[28] T. Nakano, K. Inoue, J. Hirohara et al., "Long-term prognosis of primary biliary cirrhosis (PBC) in Japan and analysis of the factors of stage progression in asymptomatic PBC (a-PBC)," Hepatology Research, vol. 22, no. 4, pp. 250-260, 2002.

[29] U. Leuschner, "Primary biliary cirrhosis-presentation and diagnosis," Clinical Liver Disease, vol. 7, no. 4, pp. 741-758, 2003.

[30] Y. Muto, S. Sato, A. Watanabe et al., "Effects of oral branchedchain amino acid granules on event-free survival in patients with liver cirrhosis," Clinical Gastroenterology and Hepatology, vol. 3, no. 7, pp. 705-713, 2005.

[31] Y. Muto, S. Sato, A. Watanabe et al., "Overweight and obesity increase the risk for liver cancer in patients with liver cirrhosis and long-term oral supplementation with branched-chain 
amino acid granules inhibits liver carcinogenesis in heavier patients with liver cirrhosis," Hepatology Research, vol. 35, no. 3, pp. 204-214, 2006.

[32] M. Kobayashi, K. Ikeda, Y. Arase et al., "Inhibitory effect of branched-chain amino acid granules on progression of compensated liver cirrhosis due to hepatitis C virus," Journal of Gastroenterology, vol. 43, no. 1, pp. 63-70, 2008.

[33] H. Fukushima, Y. Miwa, M. Shiraki et al., "Oral branched-chain amino acid supplementation improves the oxidized/reduced albumin ratio in patients with liver cirrhosis," Hepatology Research, vol. 37, no. 9, pp. 765-770, 2007.

[34] Y. Inoue, A. Koizumi, Y. Wada et al., "Risk and protective factors related to mortality from pneumonia among middleaged and elderly community residents: the JACC Study," Journal of Epidemiology, vol. 17, no. 6, pp. 194-202, 2007.

[35] G. M. Van Dam and C. H. Gips, "Primary biliary cirrhosis in The Netherlands. An analysis of associated diseases, cardiovascular risk, and malignancies on the basis of mortality figures," Scandinavian Journal of Gastroenterology, vol. 32, no. 1, pp. 77-83, 1997. 


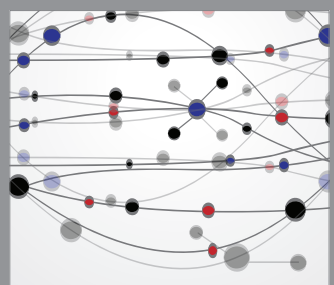

The Scientific World Journal
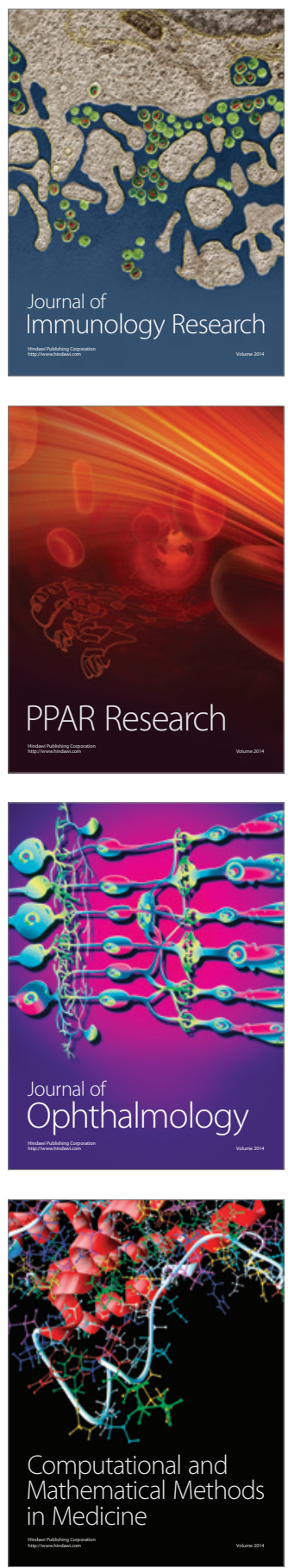

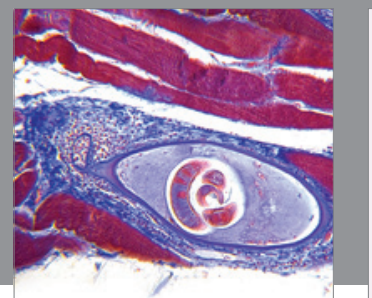

Gastroenterology

Research and Practice
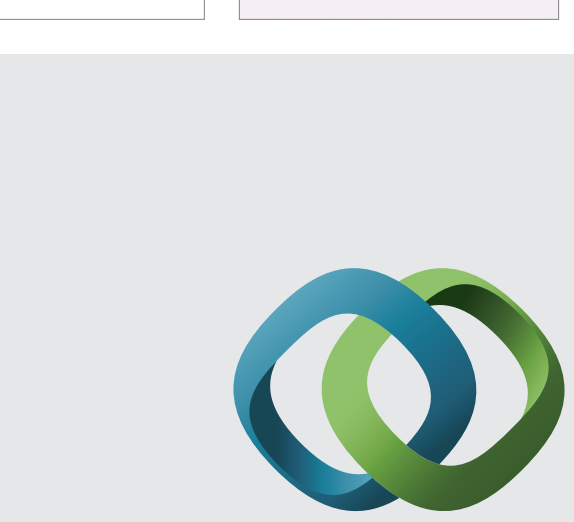

\section{Hindawi}

Submit your manuscripts at

http://www.hindawi.com
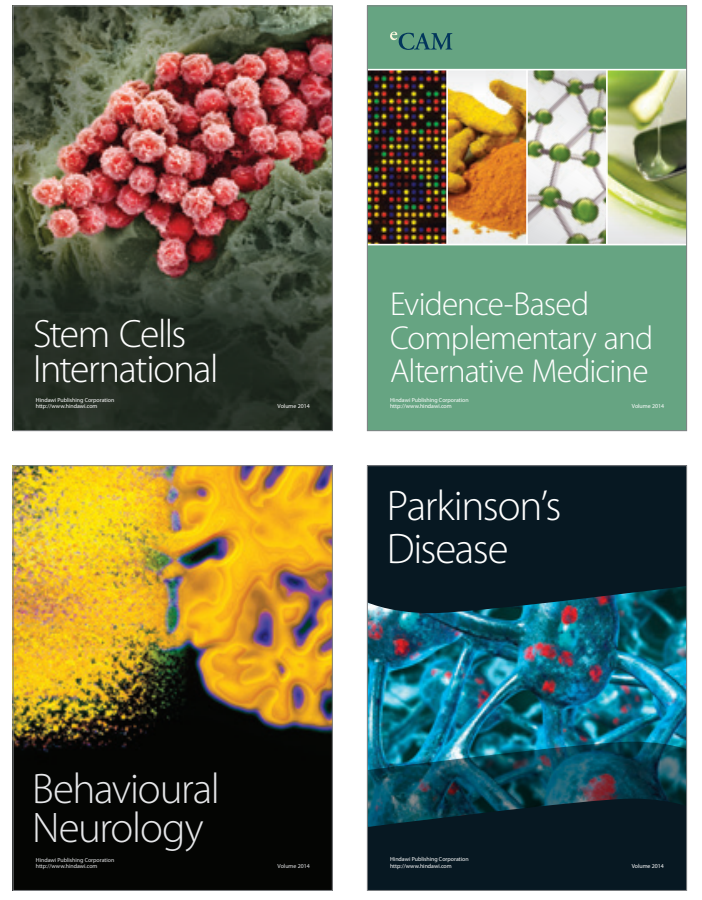
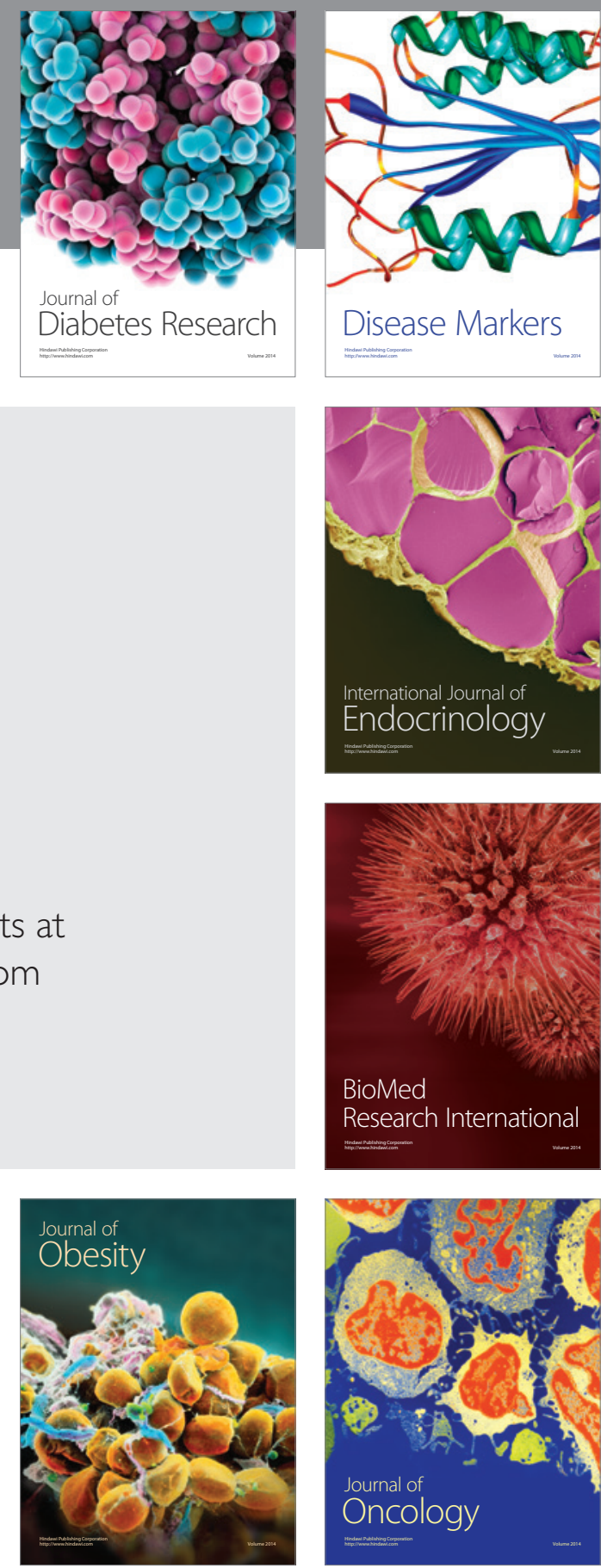

Disease Markers
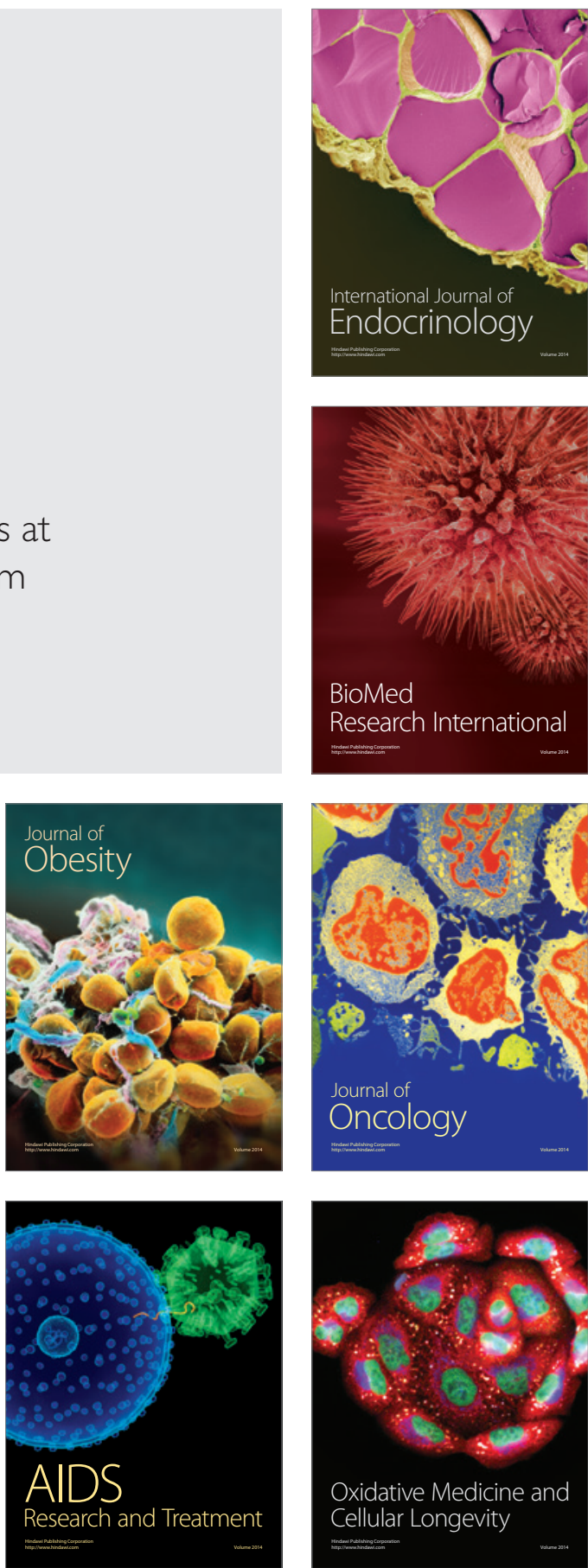